\title{
Fast Electron Beam Switching in Dielectric Samples
}

\author{
H.-J. Fitting ${ }^{1}$ and M. Touzin ${ }^{2}$ \\ ${ }^{1}$ Institute of Physics, University of Rostock, Universitaetsplatz 3, D-18051 Rostock, Germany \\ ${ }^{2}$ Laboratoire de Structure et Propriétés de l'Etat Solide, UMR CNRS 8008, \\ Université de Lille 1, F-59655 Villeneuve d'Ascq, France
}

The basic investigations of time-dependent transport are made for secondary electron (SE) relaxation and attenuation by means of Monte Carlo simulations, [1,2]. These MC calculation of ballistic electron scattering in dielectrics are based on interactions with optical and acoustic phonons as well as on impact ionization of valence band electrons, i.e. the creation of so-called tertiary electrons (TE) and cascading, see Fig. 1 to 3. The strongest cooling of SE occurs over femtoseconds and is given by the impact ionization process, whereas the electron-phonon scattering leads to slower attenuation.

The electron beam induced selfconsistent charge transport and secondary electron emission in insulators are described by means of an electron-hole flight-drift model (FDM) implemented by an iterative computer simulation, [3-5]. Ballistic secondary electrons and holes, their attenuation and drift, as well as their recombination, trapping, and field- and temperature-dependent Poole-Frenkel detrapping are demonstrated in Fig. 4.

As a main result the time dependent spatial distributions of currents $j(\mathrm{x}, \mathrm{t})$, charges $\sigma(\mathrm{x}, \mathrm{t})$, field $F(\mathrm{x}, \mathrm{t})$, as well as, the secondary electron emission rate $\sigma(\mathrm{t})$ and the surface potential $V_{0}(\mathrm{t})$ are obtained as demonstrated partially in Fig. 5. This charging process corresponds fairly to the conventional SEE resistance model discussed in more detail on the previous M\&M conference, see Ref. [6]. There a certain sample resistance $R_{\mathrm{i}}$ controls the charging of the semiconducting or semiinsulating sample and the actual landing energy $E_{0}^{\prime}$ of the electron beam is increased or diminished by the surface potential $V_{0} \gtrless 0: \quad \quad E_{0}^{\prime}=E_{0}+e V_{0}=E_{0}+e(\sigma-1) i_{0} R_{\mathrm{i}}$.

Whereas the switching-on of the secondary electron emission proceeds over milliseconds due to selfconsistent charging, see Fig. 5, the switching-off process occurs much faster, even over femtoseconds as demonstrated in Fig. 6 and will be described more detailed in Ref. [7].

Thus a rapid electron beam switching becomes possible with formation of ultra-short electron beam pulses offering an application in stroboscopic electron spectroscopy and microscopy.

\section{References:}

[1] H.-J. Fitting, E. Schreiber, J.-Ch. Kuhr, A. von Czarnowski, J. Electron Spectroscopy and Related Phenom. 119 (2001) 35-47.

[2] E. Schreiber, H.-J. Fitting, J. Electron Spectroscopy \& Rel. Phenomena 124 (2002) 25 - 37.

[3] X.Meyza, D.Goeuriot, C.Guerret-Piécourt, D.Tréheux, H.-J.Fitting, J.Appl. Phys. 8(2003)5384.

[4] M. Touzin, D. Goeuriot, C. Guerret-Piécourt, D. Juvé, D.Tréheux, H.-J. Fitting, J. Appl. Phys. 99 (2006) 114110.

[5] N. Cornet, D. Goeuriot, C. Guerret-Piécourt, D. Juvé, D.Tréheux, M. Touzin, H.-J. Fitting, J. Appl. Phys. 103 (2008) 064110.

[6] H.-J. Fitting, J. Z. Kovacs, M\&M 2009 in Richmond, Microscopy and Microanalysis 15 (Suppl.2) 2009, p. 1106 CD

[7] H.-J. Fitting, M. Touzin, J. Appl. Phys. ... (2010) submitted. 
PE and SE Trajectories

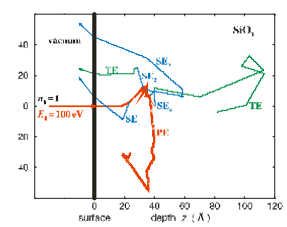

One primary electron (PE)
with SE and TE
creation

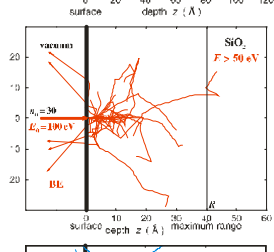
$\mathrm{eV}$

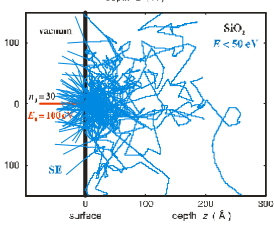

Fig. 1 Trajectories of primary (PE), secondary (SE), and tertiary (TE) electrons excited in silica with a PE incident energy $E_{0}=100 \mathrm{eV}$.

\section{Flight-Drift Model}

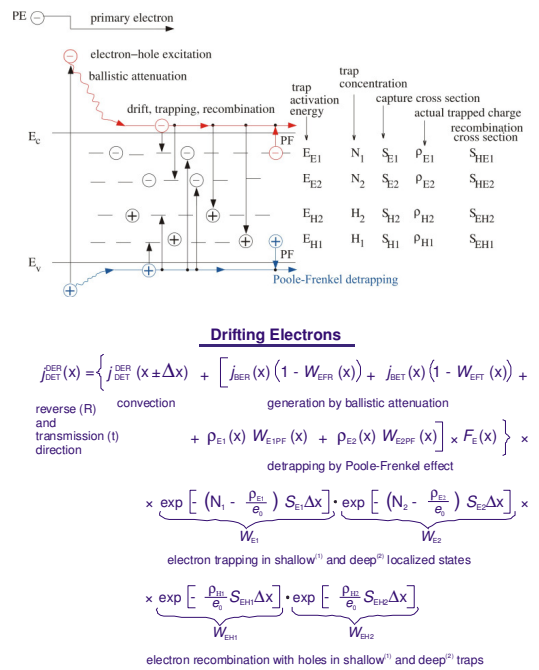

Fig.4 Scheme of the flight-drift model FDM including the ballistic flight with attenuation followed by drift, trapping, detrapping or recombination of electrons and holes.
SE Relaxation

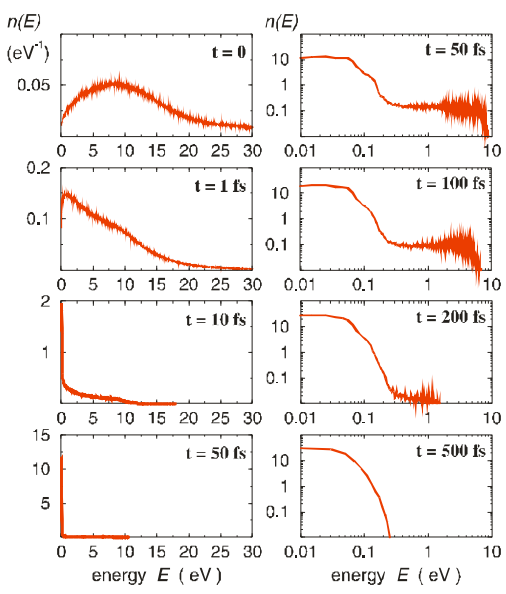

Fig. 2 Secondary electron energy relaxation $n(E)$ with time $t$ in silica. The initial rapid cooling is due to cascading.

\section{SE Switching-on}
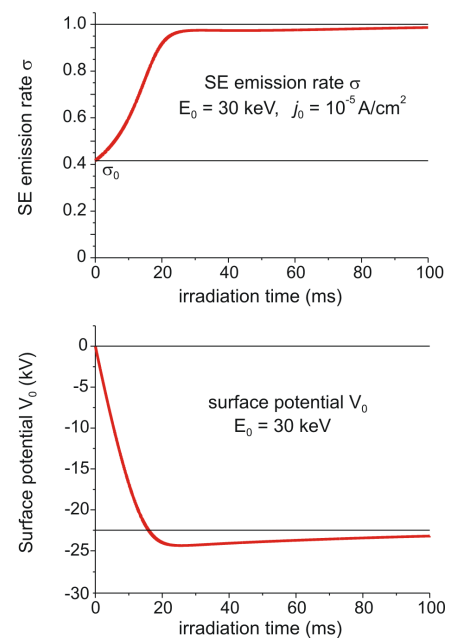

Fig.5 Overall secondary electron emission rate $\sigma$ (top) and surface potenial $V_{0}$ (below) of a bulk (3mm) alumina target as a function of irradiation time $t$.
SE Attenuation
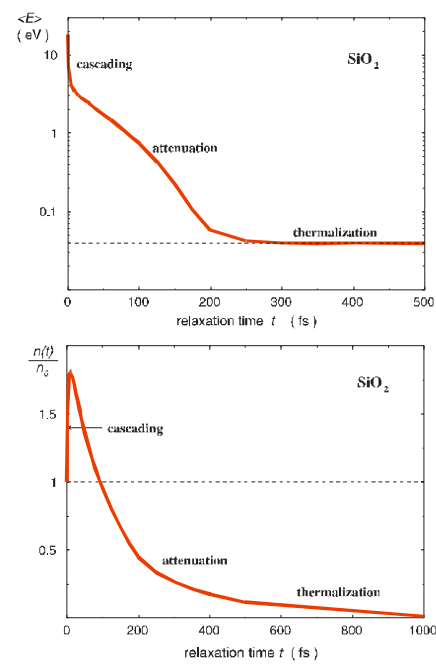

Fig.3 Mean energy $<E>$ and the number $n(t)$ of secondary electrons in silica as a function of relaxation time $t$.

\section{SE Switching-off}
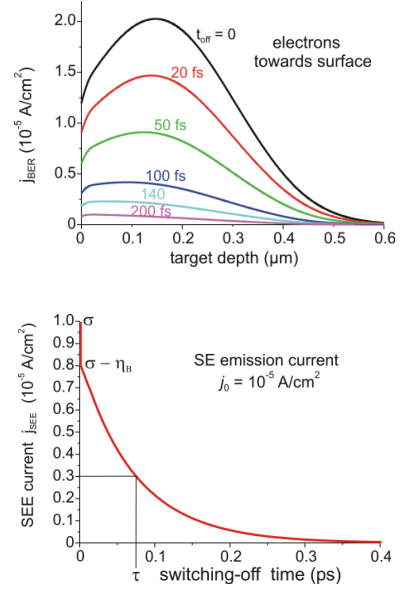

Fig.6 Switching-off process of ballistic electrons towards the surface (top) and the very rapid decay of the secondary emission rate (below). 